\title{
Fumonisin B1 Induces Apoptosis in Sphingosine 1-Phosphate Lyase-null F9 Cells through Increase of Sphingolipids Levels
}

\author{
Seon-Mi PaK ${ }^{1}$, Nam-Young ParK ${ }^{1}$, Myung-Yong PARK ${ }^{1}$, Wan-Jong Kım ${ }^{2}$, \\ Jong-Hwa LEE ${ }^{2}$, Seikwan $\mathrm{OH}^{3}$, Hwan-Soo Yoo', and Yong-Moon LEE ${ }^{1 *}$ \\ ${ }^{1}$ College of Pharmacy, Chungbuk National University, 21 Kaesin-Dong, Chongju 361-763, Korea \\ ${ }^{2}$ Department of Life Sciences, Soonchunhyang University, Asan 336-745, Korea \\ ${ }^{3}$ Department of Neuroscience, School of Medicine, Ewha Womans University, Seoul 158-710, Korea
}

(Received May 27, 2008; Accepted June 6, 2008)

\begin{abstract}
Apoptosis is essential for a variety of pathophysiological progress. Apoptosis induction by various agents changes cellular morphology, DNA content and lipid membrane composition. Recently, sphingosine 1phosphate (S1P) is avidly released from not only platelets and erythrocytes but vascular endothelium. Here we established S1P releasing cells by deleting S1P lyase (F9-12 cells). We observed apoptosis induction by the treatment of fumonisin B1 (FB1) in F9-12 cells but not in F9 wild-type cells. We measured high amounts of accumulated S1P and dihydroS1P (DHS1P) in FB1-induced apoptotic F9-12 cells. We also showed DHS1P release in an early stage of the apoptosis induction by FB1 but not by phorbol 12-myristate 13-acetate (PMA)induced apoptosis, suggesting differential apoptotic processes.
\end{abstract}

Keywords: Apoptosis, Sphingosine 1-phosphate, Release, F9 cells, HPLC

\section{INTRODUCTION}

The sphingolipids metabolites ceramide, sphingosine, and sphingosine-1-phosphate (S1P) are critical regulators in cell proliferation, survival, and apoptosis. Generally, ceramide and sphingosine inhibit proliferation and induce apoptosis, while S1P stimulates growth and suppresses apoptosis induced by pro-apoptotic signals. Because these metabolites are easily able to convert into related metabolites, it has been proposed that the relative levels of each metabolites determine cell fate (Maceyka et al., 2002). Many external stimuli activate sphingosine kinase (Sphk), leading to an increase in S1P levels (Cuvillier et al., 1996). The increased S1P may be released outside by unknown mechanisms and binds to five kinds of S1P receptors (S1P1 S1P5) to transmit the S1P signals to themselves and/or neighboring cells.

Previously, S1P is released from a variety of hematopoietic cells, including platelets (Yatomi et al., 1995), neutrophil (MacKinnon et al., 2002), erythrocytes (Hanel, Andreani, and Graler, 2007; Pappu et al., 2007) and mast

\footnotetext{
${ }^{*}$ Corresponding author

Tel: +82-43-261-2825, Fax: +82-43-268-2732

E-mail: ymleefn@chungbuk.ac.kr
}

cells (Olivera et al., 2006), astrocytes (Anelli et al., 2005). Recent data suggested vascular endothelium avidly releases high amount of S1P, considering endothelial cells as a major source of plasma S1P, showing its halflife is extremely short ( 15 min) (Venkataraman et al., 2008). Although the precise mechanism of S1P release through cellular membranes is still imaginary, some inhibitors or siRNA studies of ATP-binding cassette (ABC) transporters reduce $S 1 P$ release, indicating the involvement of various $A B C$ transporters as S1P carriers (Lee et al., 2007; Mitra et al., 2006; Sato et al., 2007).

In this study, we investigated intracellular sphingolipids accumulation and extracellular sphingolipids release in S1P lyase-null F9-12 cells which shows high sensitivity to fumonisin B1 (FB1) exposure. And it was found that S1P and dihydroS1P (DHS1P) were highly accumulated in F9-12 cells and the accumulated DHS1P were released in an early stage of the apoptosis induction by FB1.

\section{MATERIALS AND METHODS}

\section{Materials}

$D$-erythro-dihydrosphingosine (sphinganine, DHS), $D$ erythro-sphingosine, sphingosine 1-phosphate (S1P), sphinganine 1-phosphate (DHS1P), and fumonisin $B_{1}$ 
$\left(\mathrm{FB}_{1}\right)$ were purchased from Biomol Research, Inc. (Plymouth Meeting, PA., USA). Internal standards; $\mathrm{C}_{17}$-sphingosine and $\mathrm{C}_{17}$-sphingosine 1-phosphate $\left(\mathrm{C}_{17}-\mathrm{S} 1 \mathrm{P}\right)$ were obtained from Avanti Polar Lipids, Inc. (Alabaster, Al., USA). Alkaline phosphatase (APase) (No.P-6774), sodium 3'-[1-[(phenylamino)carbonyl]-3,4-tetrazolium-bis (4-methoxy-6-nitro) benzene-sulfonic acid hydrate (XTT), phenazine methosulfate (PMS) were from Sigma (St. Louis, MO., USA). Fetal bovine serum (FBS) and culture medium were obtained from Life Technologies, Inc. (Gaithersburg, MD., USA). HPLC-grade acetonitrile were purchased from Mallinckrodt Baker, Inc. (Phillipsburg, NJ., USA). o-Phthalaldehyde (OPA) was obtained from Nacalai Tesque (Kyoto, Japan). All organic solvents for sphingolipids extraction were from Merck (Darmstadt, Germany).

\section{Cell culture}

Mouse F9 teratocarcinoma cells were grown in Dulbecco's modified Eagle's medium (D6429; Sigma) containing $10 \%$ fetal calf serum supplemented with 100 units/ $\mathrm{ml}$ penicillin and $100 \mu \mathrm{g} / \mathrm{ml}$ streptomycin in $0.1 \%$ gelatincoated dishes.

\section{Production of F9-12 cells and stable transformants}

The pCE-puro HA-SPHK1a plasmid $(1 \mu \mathrm{g})$ was transfected into $4 \times 10^{5}$ F9 cells using LipofectAMINE ${ }^{\text {TM }}$ 2000 reagent. Cells were subjected to puromycin selection at $0.5 \mu \mathrm{g} / \mathrm{ml}$ for 1 week. One of the stable transformants, F9-9, expressed the highest level of HA-SPHK1a among the isolated clones and was used for further analyses. Then, The linearized targeting vector Neo $(1 \mu \mathrm{g})$ was transfected into $4 \times 10^{5}$ F9 cells using LipofectAMINE ${ }^{\text {TM }} 2000$ reagent (Invitrogen). Cells were subjected to G418 selection at $900 \mu \mathrm{g} / \mathrm{ml}$ for 1 week. Homologous recombination was examined by PCR amplification of genomic DNA using primer A (5'GTGACTTCTGGGGGAACGGAAAGC-3'), located in exon 7 but outside the genomic sequences present in the targeting vector, and primer B (5'-ATCGGAATTCCTCGAGTCTAGAGCG-3'), located upstream of the loxP site (Kihara et al., 2003).

\section{Annexin V staining assay}

Apoptotic cells was visualized under the fluorescence microscope (Carl Zeiss) by using a technique combining fluorescein (FITC)-conjugated Annexin V (Annexin VFITC) and propidium iodide (PI) staining solution (Annexin V-FITC Apoptosis Detection kit I (BD PharMingen, San Diego, CA) in accordance with the manufac- turer's instructions.

\section{S1P enrichment by immobilized metal affinity chro- matography (IMAC)}

PHOS-Select ${ }^{\mathrm{TM}}$ iron affinity gel ( $40 \mathrm{ul}$ of $50 \%$ beads slurry) was incubated with $2-5 \mathrm{ml}$ of cell culture media, containing 5-10 pmol of internal standard C17-S1P. The binding of S1P with the IMAC resin was conducted in 250 $\mathrm{mM}$ acetic acid ( $\mathrm{pH}$ 2.5-3.0) in 30\% acetonitrile for $60 \mathrm{~min}$ at $4 \mathrm{C}$. After washing the resin with $0.5 \mathrm{ml}$ of $250 \mathrm{mM}$ acetic acid ( $\mathrm{pH} 2.5-3.0)$ in 30\% acetonitrile and subsequently with $0.5 \mathrm{ml}$ of deionized water, bound S1P was eluted in $0.5 \mathrm{ml}$ of 150 or $400 \mathrm{mM}$ ammonium hydroxide in $25 \%$ acetonitrile (Lee et al., 2007).

\section{Sphingolipids analysis}

After treatment of FB1 or PMA, F9 cells and F9-12 cells (murine teratocarcinoma cells) were incubated with 2 or $4 \mathrm{ml}$ of DMEM containing $20 \mathrm{mM}$ HEPES-KOH pH 7.4, $10 \mathrm{mM}$ sodium glycerophosphate, $5 \mathrm{mM}$ sodium fluoride and $1 \mathrm{mM}$ semicarbazide and $0.5 \%$ fatty acid free BSA for trapping S1P released from the cells. Cells were incubated in the trapping medium for $2 \mathrm{~h}$. Accumulation of extracellular S1P or DHS1P was determined by spiking 5 or 10 pmol of C17-S1P to conditioned media, affinity isolation with the IMAC beads followed by the HPLC analysis of S1P or DHS1P was carried out as described previously (Min et al., 2002).

\section{RESULTS}

S1P (DHS1P) is newly synthesized from sphingosine (DHS) by sphingosine kinases. While S1P phosphatase converts S1P into sphingosine by liberating phosphate from S1P, S1P lyase disintegrates S1P into hexadecenal and phosphoethanolamine (Desai et al., 1992).

We firstly established sphingolipids-accumulating F9 cells by deleting S1P lyase (F9-12 cells) as described (Kihara et al., 2003). To obtain insight into potential significance of S1P lyase, sphingolipid metabolites, S1P, DHS1P, sphingosine and DHS were measured.

The F9-12 $\left(\mathrm{SPL}^{-1-}\right)$ cells became highly sensitive to FB1 and high rate of cell death was observed, while the F9 $\left(\mathrm{SPL}^{+/+}\right)$cells were not so strongly affected in the same condition. By treatment of high concentration FB1 for $48 \mathrm{~h}$, high population of Annexin V stained F9-12 cells were observed (Fig. 1). Therefore, the deletion of S1P lyase function made cells to be sensitive to FB1-induced apoptosis, suggesting possible involvement of accumulated sphingolipids on the apoptosis induction. 


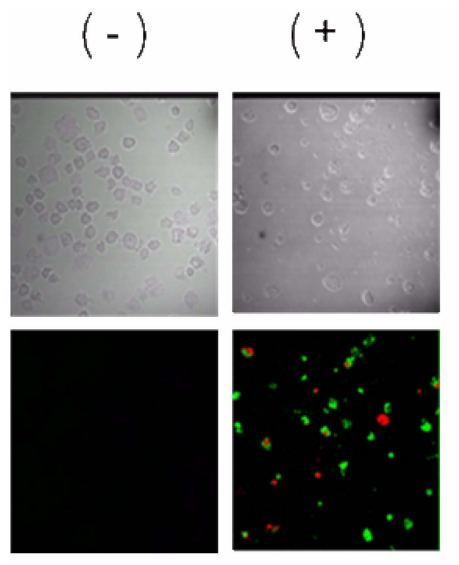

F9-12 cells (S1P lyase KO)
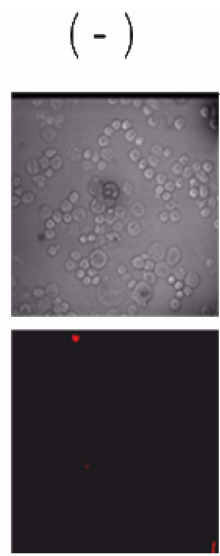

F9 cells (S1P lyase WT)
$(+) \quad$ FB1

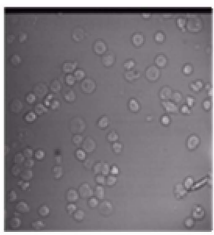

Phase Contrast

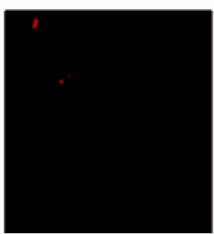

Annexin $\mathrm{V}+\mathrm{Pl}$

Fig. 1. FB1 treatment (50 uM) for $48 \mathrm{~h}$ induced apoptotic cells only in F9-12 cells, not in F9 cells. Annexin V-FITC fluorescence was largely observed, suggesting those cells were apoptotic cells. Cells were harvested and incubated with Annexin V and propidium iodide for $1 \mathrm{~h}$ before microscopic observation.

Assuming that the apoptosis by FB1 was induced by abnormal accumulation of sphingolipids, we measured the levels of sphingosine, DHS, S1P and DHS1P both in the F9-12 cells and the F9 cells (wild type) after FB1 treatment for $48 \mathrm{~h}$. Unexpectedly, the amounts of S1P and DHS1P in the F9-12 cells were obviously raised up over hundred times compared with those from the wildtype F9 cells (Fig. 2A and B). As expected from the fact of that FB1 specifically inhibits ceramide synthase, DHS and sphingosine was also increased both in the $\mathrm{F9}$ and the F9-12 cells (Fig. 3A and B). Considering that sphingosine and DHS are pro-apoptotic and S1P and DHS1P are anti-apoptotic, these results might suggest that accu-
A

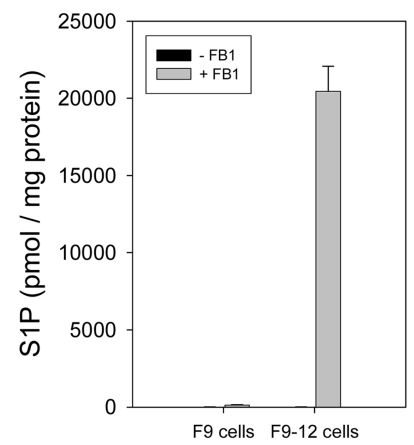

B

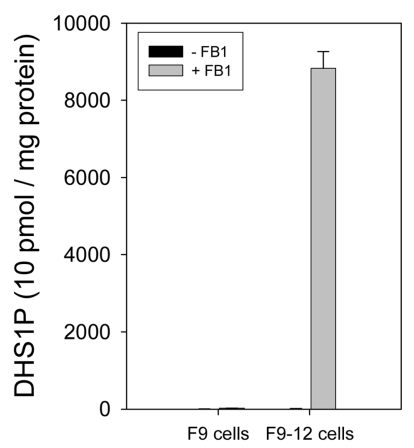

Fig. 2. FB1 treatment (50 uM) for $48 \mathrm{~h}$ greatly accumulates S1P (A) and DHS1P (B). The sphingolipids measurement by HPLC was an indirect detection of sphingosine and DHS after dephosphorylation of S1P and DHS1P by alkaline phosphatase described in Material and Methods. C17-S1P (20 100 pmol) was used as an internal standard. mulated DHS and sphingosine induced apoptosis. For the anti-apoptotic action of S1P/DHS1P, they should be released into extracellular media and act on S1P receptors in the plasma membrane.

Next, we measured amounts of DHS and DHS1P released from F9-12 cells after FB1 (50 uM) treatment for $8 \mathrm{~h}$. During this early stage, there were a little sign of apoptosis induction (data not shown). The collected conditioned media were incubated with IMAC resin to enrich S1P and DHS1P in the media. In the early apoptosis stage, significant amount of DHS1P were already observed in F9-12 cells, indicating that DHS1P once endogenously synthesized by FB1 treatment was readily
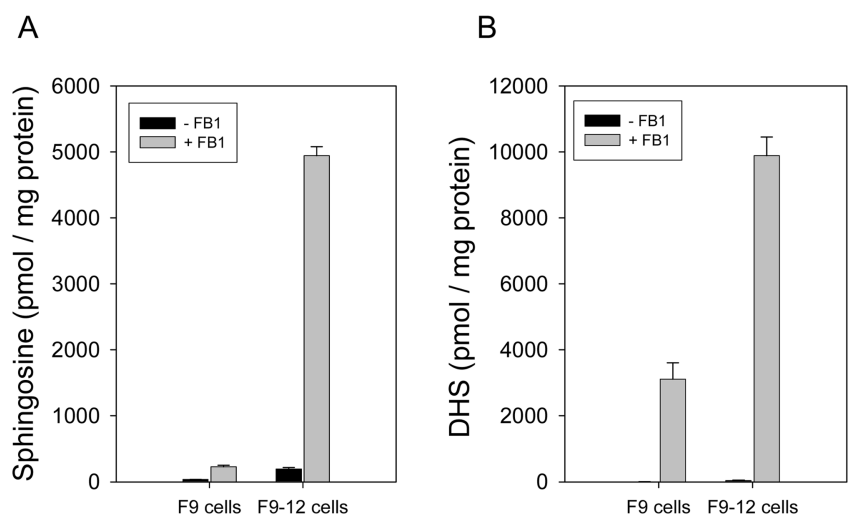

Fig. 3. Nave F9-12 cells have higher S1P levels (A). FB1 treatment increases sphingosine and greatly enhanced cellular DHS levels in F9-12 cells and less in F9 cells (B). C17-sphingosine (30 100 pmol) was used as an internal standard. 
A

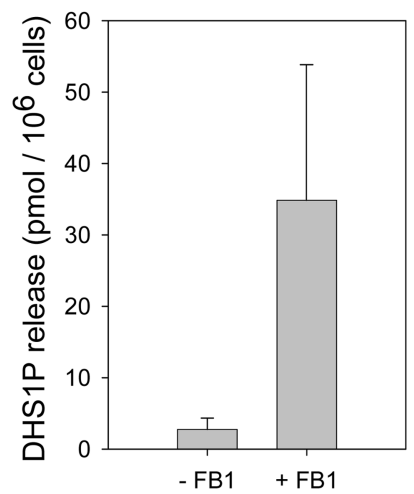

B

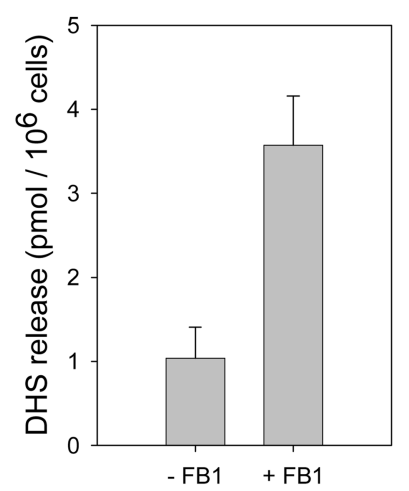

Fig. 4. FB1 treatment $(50 \mathrm{uM})$ for $8 \mathrm{~h}$ enter the F9-12 cells into early apoptotic stage. However, the release of DHS1P $(A)$ and less DHS (B) was observed, indicating that the beginning for the release of DHS1P occur in very early stage in apoptosis.

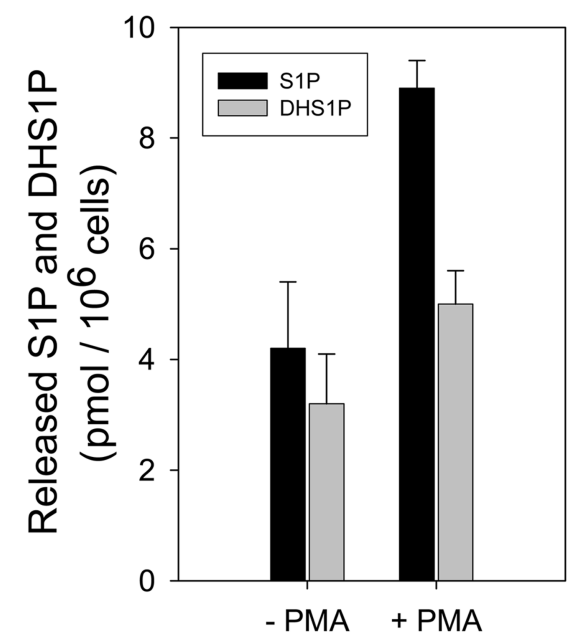

Fig. 5. PMA treatment $(100 \mathrm{nM})$ for $12 \mathrm{~h}$ in F9-12 cells slightly increased the S1P levels. In this condition, there were no apoptotic cells. Unlike FB1 treatment, PMA did not greatly increase the DHS1P levels, indicating sphingolipids release was dependent on types of proapoptotic agents.

released outside the cells (Fig. 4A). However, the release of DHS was not observed although the DHS was quickly accumulated by FB1 treatment in F9-12 cells (Fig. 4B). Interestingly, the release of S1P and sphingosine was not significantly observed in this condition (data not shown).

Alternatively, we also confirmed the concomitant increment of S1P and DHS1P levels from the cultured media with $100 \mathrm{nM}$ PMA treatment for $12 \mathrm{~h}$ (Fig. 5).

\section{DISCUSSION}

The F9-12 cells were extraordinarily sensitive to FB1

exposure compared to F9 wild-type cells, suggesting that the loss of S1P lyase function was a critical factor on the accumulation of sphingolipids such as S1P or DHS1P and apoptosis induction (Fig. 2A and B). Indeed, inhibition of S1P lyase induces lymphocyte sequestration and disruption of S1P gradients (Schwab et al., 2005). Thus, F9-12 cells (S1P lyase null) with FB1 could be a model S1P-releasing cell system to observe the role of S1P lyase in the clearance and release of S1P.

Fumonisins are a family of mycotoxins produced by Fusarium verticillioides. Most of FB1 toxicities can be explained by its ability to alter sphingolipids metabolism by inhibiting ceramide synthase. At least, the elevation in DHS mediates the earliest toxicity of FB1 (Merrill et al., 1993). Recent studies suggested the possible role of DHS1P sometimes elevated by Sphk1 in cell- or tissue specific manners (Berdyshev et al., 2006; Kariya et al., 2005).

Here, we firstly demonstrated DHS1P release by FB1 treatment in S1P lyase-null cells, suggesting that extracellular DHS1P released from FB1-treated cells could act through cell surface S1P receptors. On the other hand, the S1P release by PMA treatment may suggest differential regulation of sphingolipids accumulation and release by different apoptotic stimuli.

Taken together, we quantitatively measured the released S1P and DHS1P from F9-12 cells, in which cells are sensitive to pro-apoptotic agents. S1P lyase may play a key role to regulate cellular S1P levels and therefore its release into media.

\section{ACKNOWLEDGEMENTS}

This work was supported by the research grant of the Chungbuk National University in 2006.

\section{REFERENCES}

Anelli, V., Bassi, R., Tettamanti, G., Viani, P., and Riboni, L. (2005). Extracellular release of newly synthesized sphingosine-1-phosphate by cerebellar granule cells and astrocytes. J. Neurochem. 92(5), 1204-1215.

Berdyshev, E. V., Gorshkova, I. A., Usatyuk, P., Zhao, Y., Saatian, B., Hubbard, W., and Natarajan, V. (2006). De novo biosynthesis of dihydrosphingosine-1-phosphate by sphingosine kinase 1 in mammalian cells. Cell Signal 18(10), 1779-1792.

Cuvillier, O., Pirianov, G., Kleuser, B., Vanek, P. G., Coso, O. A., Gutkind, S., and Spiegel, S. (1996). Suppression of ceramide-mediated programmed cell death by sphingosine-1-phosphate. Nature 381(6585), 800-803.

Desai, N. N., Zhang, H., Olivera, A., Mattie, M. E., and Spiegel, S. (1992). Sphingosine-1-phosphate, a metabolite of sphin- 
gosine, increases phosphatidic acid levels by phospholipase D activation. J. Biol. Chem. 267(32), 23122-23128.

Hanel, P., Andreani, P., and Graler, M. H. (2007). Erythrocytes store and release sphingosine 1-phosphate in blood. Faseb. J. 21(4), 1202-1209.

Kariya, Y., Kihara, A., Ikeda, M., Kikuchi, F., Nakamura, S., Hashimoto, S., Choi, C. H., Lee, Y. M., and Igarashi, Y. (2005). Products by the sphingosine kinase/sphingosine 1 phosphate (S1P) lyase pathway but not S1P stimulate mitogenesis. Genes Cells 10(6), 605-615.

Kihara, A., Ikeda, M., Kariya, Y., Lee, E. Y., Lee, Y. M., and Igarashi, Y. (2003). Sphingosine-1-phosphate lyase is involved in the differentiation of F9 embryonal carcinoma cells to primitive endoderm. J. Biol. Chem. 278(16), 14578-14585.

Lee, Y. M., Venkataraman, K., Hwang, S. I., Han, D. K., and Hla, T. (2007). A novel method to quantify sphingosine 1-phosphate by immobilized metal affinity chromatography (IMAC). Prostaglandins Other Lipid Mediat 84(3-4), 154-162.

Maceyka, M., Payne, S. G. Milstien, S., and Spiegel, S. (2002). Sphingosine kinase, sphingosine-1-phosphate, and apoptosis. Biochim. Biophys. Acta 1585(2-3), 193-201.

MacKinnon, A. C., Buckley, A., Chilvers, E. R., Rossi, A. G., Haslett, C., and Sethi, T. (2002). Sphingosine kinase: a point of convergence in the action of diverse neutrophil priming agents. J. Immunol 169(11), 6394-6400.

Merrill, A. H., Jr., van Echten, G., Wang, E., and Sandhoff, K. (1993). Fumonisin B1 inhibits sphingosine (sphinganine) Nacyltransferase and de novo sphingolipid biosynthesis in cultured neurons in situ. J. Biol. Chem. 268(36), 27299-27306.

Min, J. K., Yoo, H. S., Lee, E. Y., Lee, W. J., and Lee, Y. M. (2002). Simultaneous quantitative analysis of sphingoid base 1-phosphates in biological samples by o-phthalaldehyde precolumn derivatization after dephosphorylation with alkaline phosphatase. Anal. Biochem. 303(2), 167-175.

Mitra, P., Oskeritzian, C. A., Payne, S. G., Beaven, M. A., Milstien, S., and Spiegel, S. (2006). Role of ABCC1 in export of sphingosine-1-phosphate from mast cells. Proc. Natl. Acad Sci. USA 103(44), 16394-16399.

Olivera, A., Urtz, N., Mizugishi, K., Yamashita, Y., Gilfillan, A. M., Furumoto, Y., Gu, H., Proia, R. L., Baumruker, T., and Rivera, J. (2006). IgE-dependent activation of sphingosine kinases 1 and 2 and secretion of sphingosine 1-phosphate requires Fyn kinase and contributes to mast cell responses. J. Biol. Chem. 281(5), 2515-2525.

Pappu, R., Schwab, S. R., Cornelissen, I., Pereira, J. P., Regard, J. B., Xu, Y., Camerer, E., Zheng, Y. W., Huang, Y., Cyster, J. G., and Coughlin, S. R. (2007). Promotion of lymphocyte egress into blood and lymph by distinct sources of sphingosine-1-phosphate. Science 316(5822), 295-298.

Sato, K., Malchinkhuu, E., Horiuchi, Y., Mogi, C., Tomura, H., Tosaka, M., Yoshimoto, Y., Kuwabara, A., and Okajima, F. (2007). Critical role of ABCA1 transporter in sphingosine 1phosphate release from astrocytes. J. Neurochem. in press.

Schwab, S. R., Pereira, J. P., Matloubian, M., Xu, Y., Huang, Y., and Cyster, J. G. (2005). Lymphocyte sequestration through S1P lyase inhibition and disruption of S1P gradients. Science 309(5741), 1735-1739.

Venkataraman, K., Lee, Y. M., Michaud, J., Thangada, S., Ai, Y., Bonkovsky, H. L., Parikh, N. S., Habrukowich, C., and Hla, T. (2008). Vascular endothelium as a contributor of plasma sphingosine 1-phosphate. Circ. Res. 102(6), 669-676.

Yatomi, Y., Ruan, F., Hakomori, S., and Igarashi, Y. (1995). Sphingosine-1-phosphate: a platelet-activating sphingolipid released from agonist-stimulated human platelets. Blood 86(1), 193-202. 